\title{
Role of Drift wave turbulence in amplification of high frequency waves in inhomogeneous plasma
}

\section{Mahinder Singh}

\section{International Journal of Advanced Multidisciplinary Research}

ISSN: 2393-8870

www.ijarm.com

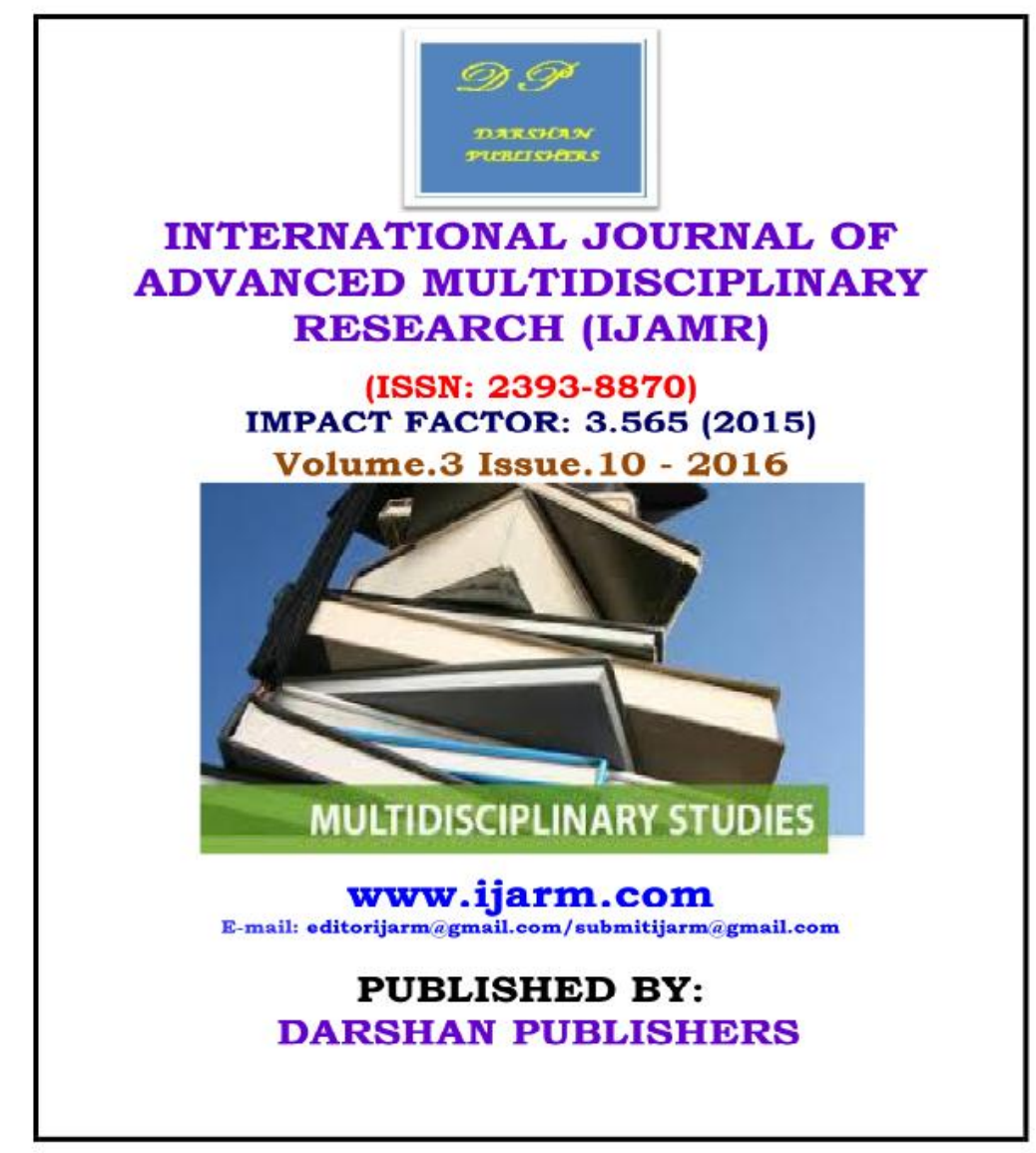

Darshan Publishers, Tamil Nadu, India 


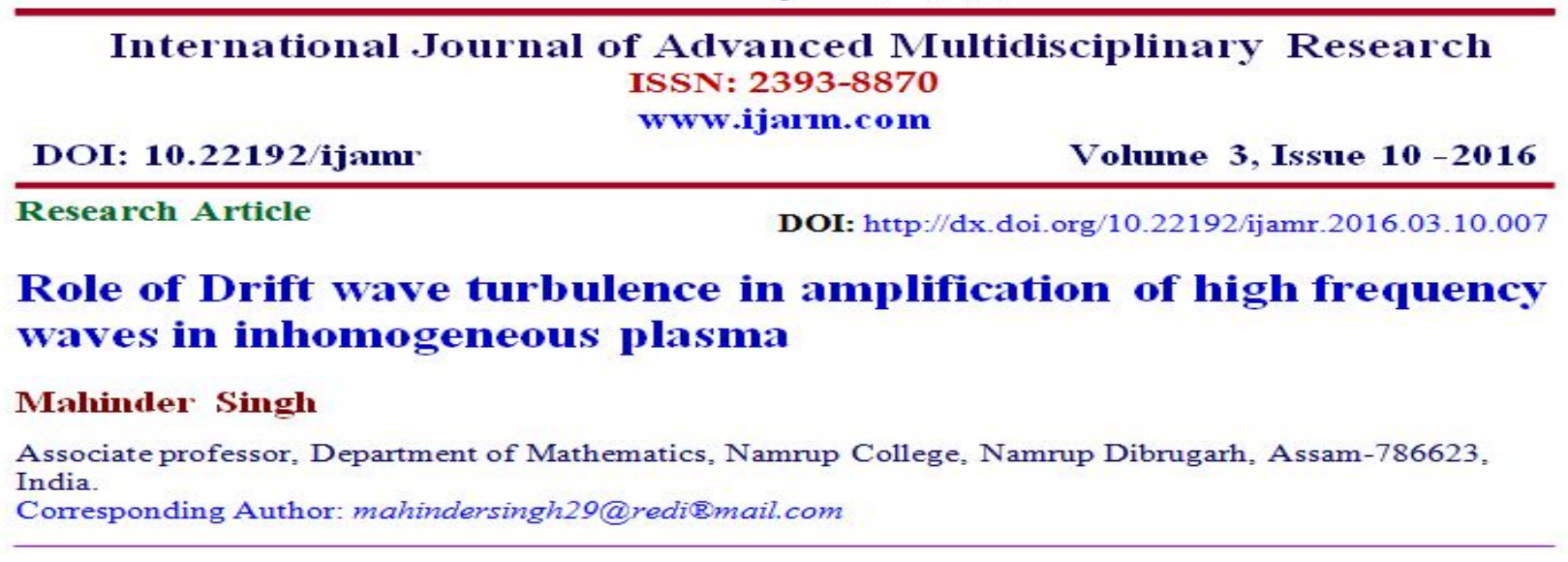

\section{ABSTRACT :}

The study on the role of drift wave turbulence associated with plasma instabilities for the generation of various unstable radiations is an interesting issue. A theoretical study on the role of drift wave turbulence, in amplification of high frequency wave has been mede on the basis of a nonlinear wave particle interaction with drift wave turbulence in an inhomogeneous plasma. Considering a Maxwellian distribution function model for inhomogeneous plasma under the standard local approximation, we have estimated the growth rate of Bernstein mode wave, which is obtained by using the nonlinear dispersion relation, It has been found that amplification of Bernstein mode wave is possible at the expense of drift wave turbulent energy, because plasma particles, acceleraed by drift wave turbulent field, may transfer their energy to Bernstein mode wave ththrough a modulated field. This result may be particularly important for stability of various drift modes in magnetically confined plasma as well as for transport of momentum and energy in such inhomogeneous systems.

\section{Introduction :}

Drift waves occupy a special place in the spectrum of collective plasma processes. Drift waves are supported by particle drifts, which arises due to gradient in density or temperature present in the system. Drift wave instabilities are inevitable in any confined plasma. The study on the role of drift wave turbulence associated with plasma instabilities for the generation of various unstable radiations is an interesting issue for plasma physicists. The earliest observations of the drift wave were made by Angelo et al.[1] and by Chen [2]. Subsequently, it has been observed that drift wave turbulence is one of the dominating turbulence wave energy available in any magneticallyconfined plasma and it is now considered as one of the potentially dangerous source of insatbility in tokamak plasma [3]. They occur in the edge and core regions of tokamak plasma [1].

Earlier efforts had been made to explain the observed auroral radiation phenomena on the basis of cyclotron maser instability, which included the effect of inhomogeneity along the geometric field lines $[4,5]$. The space craft observations provided the evidence that large and small amplitude perturbations of the drift waves associated with the kinetic Alfven wave turbulence are permanently present in the near earth plasma environment [6]. 
The low frequency drift wave fields are found to be strongly in phase relation with thermal plasma particles. Such a drift wave field may transfer their wave energy to a nonresonant wave through nonlinear wave-particle interaction process $[7,8]$.

In a nonlinear numerical simulation study [9] on collisionless drift wave turbulence, using a hybrid model of drift kinetic electrons and cold ions in three dimensional shared slab geometry, it has been observed that turbulent particle flux is directed up-gradient (inward) for sufficiently high value of $\left(\eta_{e}=\frac{L_{n}}{L_{T_{e}}}\right)$. Such particles pinch can be attributed to the completely different perpendicular dynamics of slow (resonant) and fast (nonresonant) electrons, making it a genuinely kinetic effect (different regions of electron parallel velocity $\left(w_{\|}\right)$have different dynamics). This observation suggests applicability of kinetic approach in drift wave turbulent energy exchange process.

In this paper, we are considering nonlinear wave-particle interaction process in presence of drift wave turbulence, on the baiss of new plasma turbulence theory. The new plasma turbulence theory [10 - 12] predicted a wave -particle interaction process called plasma maser effect, which makes a system enable for transfer of wave energy from the low frquency mode to the high frequency mode. Plasma-maser interaction is a nonlinear mode - mode coupling process between two types of waves in a turbulent plasma [3]. The first type of plasma wave is the low-frequency resonant mode wave and the second type is the high-frequency non-resonant mode wave. The resonant waves are those for which the Cherenkov resonance condition $\omega-\vec{k} \cdot \vec{v}=0$ is satisfied, whereas the non-resonant waves are those for which both Cherenkov condition and the nonlinear scattering conditions are not satisfied, i.e. $\Omega-\vec{K} \cdot \vec{v} \neq 0$ and $(\Omega-\omega)-(\vec{K}-\vec{k}) \cdot \vec{v} \neq 0$. Here $\omega$ and $\Omega$ are frequencies of the resonant and non-resonant waves, respectively, and $\vec{k}$ and $\vec{K}$ are the corresponding wave numbers. As a result of plasma maser effect transfer of the wave energy from lowfrequency resonant mode to the high-frequency non-resonant mode may be possible [13]. It has been theoretically established that, plasma maser effect is found to be effective in an open system, where free energy from external sources are available [14] in the form of external magnetic field. Since plasma maser effect is energy up conversion of low-frequency turbulence to high-frequency modes, it plays an important role in space plasma and as an effective radiation mechanism in the magnetosphere plasma.

In almost all the studies in plasma maser effect have been carried out considering the the plasma system as homogeneous $[15,16]$, recently attempts have been to investigate the role of density gradient parameter in energy upconversion process through plasma maser effect in inhomogeneous plasma $[7,8$ 17,18,19]. In our investigation, we are considering interaction of the drift wave turbulence present in inhomogeneous plasma with Bernstein mode wave. Considering a Maxwellian model distribution function under standard local approximation for inhomogeneous plasma [20], we have obtained the growth rate for Bernstein mode wave using nonlinear dispersion relation. Here we consider Fourier transform 
method. The real plasma, particularly in nature, is far from thermal equilibrium state. In such case, the initial value analysis is not valid [21], because the plasma is turbulent at the initial time and accordingly, the steady turbulent state is likely to be maintained by boundary conditions on past history of a plasma. The disribution function is far from a thermal equilibrium Maxwellian and the disrtibution is approximately steady within the time scale considered. Further in steady turbulent state of plasma, the fluctuation is enhanced where the disribution function is far from the thermal equilibrium state.

In our study it is found that amplification of Bernstein mode wave is possible at the expense of drift wave turbulence energy. This result is mainly important in stabilizing various drift modes in fusion plasma as well as explaining transport of momentum and energy in such inhomogeneous system of finite extent. Moreover, it is also important as nonlinear interaction of low frequecy resonant mode with thermal particles in long spatial lengths in magnetosphere can trasfer wave energy burst in space [22].

\section{Formulation:}

We consider a magnetically confined plasma in presence of drift wave turbulence. The Bernstein mode, present in the system, is considered as super imposing perturbation field to the system. The confining magnetic field with negligibly small gradient is taken along the $\vec{z}$ direction $\vec{B}_{0}=B_{0}(y)$ and the system has spatial gradient in y-direction. For such an inhomogeneous system [23] the particle distribution function is considered as

$$
f_{0 e}\left(v_{\perp}, v_{\|}, y+\frac{v_{x}}{\Omega_{e}}\right) \simeq f_{0 e}(\vec{v}, y)+\frac{v_{x}}{\Omega_{e}} \frac{\partial f_{0 e}(\vec{v}, y)}{\partial y}
$$

where $f_{0 e}(\vec{v}, y)$ is the distribution function for guiding center, $v_{x}=v_{\perp} \cos \phi, v_{\perp}$ and $v_{\|}$are components of velocity along and perpendicular direction of the external magnetic field, $\phi$ is the phase angle of the particle in the orbit and $\Omega_{e}=e B_{0} / m c$ is the electron cyclotron frequency.

We now introduce the density gradient as

$$
\epsilon^{\prime}=\left[\frac{1}{f_{0 e}} \frac{\partial f_{0 e}(\vec{v}, y)}{\partial y}\right]_{y=0}
$$

Thus the particle distribution function Eq. (1) reduces to the form

$$
f_{0 e}\left(v_{\perp}, v_{\|}, y+\frac{v_{x}}{\Omega_{e}}\right) \simeq f_{0 e}(\vec{v}, y)\left\{1+\frac{v_{x}}{\Omega_{e}} \epsilon^{\prime}\right\}
$$

The interaction of high frequency Bernstein-mode wave with low frequency drift wave turbulence is governed by Vlasov- Poisson equations

$$
\left[\frac{\partial}{\partial t}+\vec{v} \cdot \frac{\partial}{\partial \vec{r}}-\frac{e}{m}\left(\vec{E}+\frac{\vec{v} \times \vec{B}}{c}\right) \cdot \frac{\partial}{\partial \vec{v}}\right] F_{o e}(\vec{r}, \vec{v}, t)=0
$$


and

$$
\vec{\nabla} \cdot \vec{E}=4 \pi e n_{e} \int f(\vec{r}, \vec{v}, t) d v .
$$

According to the linear response theory of turbulent plasma [24], the unperturbed electron distribution function and fields are

$$
F_{0 e}=f_{0 e}+\epsilon f_{1 e}+\epsilon^{2} f_{2 e} .
$$

and

$$
\vec{E}_{0 e}=\epsilon \vec{E}_{l}+\epsilon^{2} \vec{E}_{2}
$$

where $\epsilon(<<1)$ is a small parameter associated with turbulent field $\vec{E}_{l}=\left(E_{l \perp}, 0, E_{l \|}\right)$ with propagation wave vector $\vec{k}=\left(k_{\perp}, 0, k_{\|}\right), f_{0 e}$ is space and time average part, $f_{1 e}$, $f_{2 e}$ are fluctuating parts of the distribution function and $E_{2}$ is the second order electric field.

To the order of $\epsilon$ from Eq.(3), we have

$$
\left[\frac{\partial}{\partial t}+\vec{v} \cdot \frac{\partial}{\partial \vec{r}}-\frac{e}{m}\left(\frac{\vec{v} \times \vec{B}_{0}}{c}\right) \cdot \frac{\partial}{\partial \vec{v}}\right] f_{1 e}=\frac{e}{m}\left(\vec{E}_{l} \cdot \frac{\partial}{\partial \vec{v}} f_{o e}\right) .
$$

Using Fourier transforms according to

$$
A(\vec{r}, \vec{v}, t)=\sum_{\vec{k}, \omega} A(\vec{k}, \omega) \exp [i(\vec{k} \cdot \vec{r}-\omega t)]
$$

For low-frequency electrostatic waves, the electron motion along the magnetic field is important. The Fourier component of the corresponding distribution function $f_{1 e}(\vec{k}, \omega)$, from Eq.(8), is given by

$$
f_{1 e}(\vec{k}, \omega)=\left(\frac{i e}{m}\right) \frac{E_{l \|}(\vec{k}, \omega) \frac{\partial}{\partial v_{\|}} f_{o e}}{\omega-k_{\|} v_{\|}+i 0} .
$$

where $i 0$ is the small imaginary part associated with $\omega$ and $\vec{k}$ is the wave number of Langmuir wave field.

To this quasisteady state, we apply purturbation $\mu \delta \vec{E}_{h}$ of test high-frequency Bernstein mode wave with a propagating vector $\vec{K}=\left(K_{\perp} 0,0\right)$, electric field $\delta \vec{E}=\left(\delta E_{h}, 0,0\right)$ and a frequency $\Omega$. Thus the total perturbed electric field, magnetic field and the electron distribution function are

$$
\begin{gathered}
\delta \vec{E}=\mu \delta \vec{E}_{h}+\mu \epsilon \delta \vec{E}_{l h}+\mu \epsilon^{2} \Delta \vec{E}, \\
\delta \vec{B}=0
\end{gathered}
$$




$$
\delta f=\mu \delta f_{h}+\mu \epsilon \delta f_{l h}+\mu \epsilon^{2} \Delta f .
$$

where $\delta \vec{E}_{l h}, \Delta \vec{E}$ are the modulated electric fields, $\delta f_{h}$ is the fluctuating part due to high frequency Bernstein mode wave and $\delta f_{l h}, \Delta f$ are electron distribution functions corresponding to modulated fields. In Eqs. (11) to (13), we have omitted second order field quantities, which can be justified under random phase approximations.

To the order $\mu, \mu \epsilon, \mu \epsilon^{2}$, we obtain from (4)

$$
\begin{gathered}
P \delta f_{h}=\frac{e}{m} \delta \vec{E}_{h} \cdot \frac{\partial}{\partial \vec{v}} f_{o e} . \\
P \delta f_{l h}=\frac{e}{m} \vec{E}_{l} \cdot \frac{\partial}{\partial \vec{v}} \delta f_{h}+\frac{e}{m} \delta \vec{E}_{h} \cdot \frac{\partial}{\partial \vec{v}} f_{1 e}+\frac{e}{m} \delta \vec{E}_{l h} \cdot \frac{\partial}{\partial \vec{v}} f_{o e} . \\
P \Delta f=\frac{e}{m} \vec{E}_{l} \cdot \frac{\partial}{\partial \vec{v}} \delta f_{l h}+\frac{e}{m} \delta \vec{E}_{l h} \cdot \frac{\partial}{\partial \vec{v}} f_{1 e} .
\end{gathered}
$$

where the operator $\mathrm{P}$ is given by

$$
P=\frac{\partial}{\partial t}+\vec{v} \cdot \frac{\partial}{\partial \vec{r}}-\frac{e}{m}\left(\frac{\vec{v} \times \vec{B}_{o}}{c}\right) \cdot \frac{\partial}{\partial \vec{v}} .
$$

According to method of characteristics [20], which is also known as "integration over unperturbed orbit" We solve these differential equations for the fluctuating parts of the distribution function $\delta f_{h}, \delta f_{l h}$ and $\Delta f$ over the electron trajectories. Using the Fourier transform of Eq.(9) and integrating along the unperturbed orbits we evaluate the various perturbed distribution functions from Eq.(14) to (16) to obtain the non-linear dielectric function of the Bernstein mode wave of frequency $\Omega$, in the presence of the drift wave turbulence.

Now we calculate modulated field $\delta E_{l h}(\vec{K}-\vec{k}, \Omega-\omega)$ by Poisson's equation,

$$
\nabla \cdot \delta \vec{E}_{l h}=-4 \pi e n_{e} \int \delta f_{l h} d \vec{v} .
$$

in the form 


$$
\begin{aligned}
& \delta E_{l h}(\vec{K}-\vec{k}, \Omega-\omega)=-\frac{\omega_{p e}^{2}}{|\vec{K}-\vec{k}| R} \frac{\delta E_{h}}{K_{\perp}} \int\left[\left\{\frac { i e } { m } \frac { m } { T _ { e } } \left(1+\left\{\Omega-\frac{\epsilon^{\prime} T_{e} K_{\perp}}{m \Omega_{e}}\right\}\right.\right.\right. \\
& \left.\left.\sum_{a, b} \frac{J_{a}(\alpha) J_{b}(\alpha) \exp [i(b-a) \theta]}{a \Omega_{e}-\Omega}\right)\right\} \times \\
& \left\{\frac { E _ { l \perp } } { K _ { \perp } } \frac { m } { T _ { e } } \left(1+\left\{\Omega-\omega+k_{\|} v_{\|}-\frac{\epsilon^{\prime} T_{e} K_{\perp}}{m \Omega_{e}}\right\} \times\right.\right. \\
& \left.\sum_{p, q} \frac{J_{p}\left(\alpha^{\prime}\right) J_{q}\left(\alpha^{\prime}\right) \exp [i(q-p) \theta]}{p \Omega_{e}-\Omega-k_{\|} v_{\|}+\omega}\right)- \\
& \left.E_{l \|} \frac{\partial}{\partial v_{\|}} \sum_{p, q} \frac{J_{p}\left(\alpha^{\prime}\right) J_{q}\left(\alpha^{\prime}\right) \exp [i(q-p) \theta]}{p \Omega_{e}-\Omega-k_{\|} v_{\|}+\omega}\right\}+ \\
& \frac{m}{T_{e}}\left(\frac{i e}{m} \frac{E_{l \|} \frac{\partial}{\partial v_{\|}} f_{0 e}}{\omega-k_{\|} v_{\|}+i 0}\right) \times \\
& \left(1+\left\{\Omega-\omega+k_{\|} v_{\|}-\frac{\epsilon^{\prime} T_{e} K_{\perp}}{m \Omega_{e}}\right\} \times\right. \\
& \left.\left.\sum_{p, q} \frac{J_{p}\left(\alpha^{\prime}\right) J_{q}\left(\alpha^{\prime}\right) \exp [i(q-p) \theta]}{p \Omega_{e}-\Omega-k_{\|} v_{\|}+\omega}\right)\right] d \vec{v} . \\
& R=1+\frac{4 \pi e^{2} n_{e}}{m|\vec{K}-\vec{k}|} \frac{m}{T_{e}} \int\left[\left(1+\left\{\Omega-\omega+k_{\|} v_{\|}-\frac{\epsilon^{\prime} T_{e} K_{\perp}}{m \Omega_{e}}\right\}\right.\right. \\
& \left.\sum_{a, b} \frac{J_{a}\left(\alpha^{\prime}\right) J_{b}\left(\alpha^{\prime}\right) \exp [i(b-a) \theta]}{a \Omega_{e}-\Omega-k_{\|} v_{\|}+\omega}\right)-k_{\|} \frac{\partial}{\partial v_{\|}} \times \\
& \left.\sum_{a, b} \frac{J_{a}\left(\alpha^{\prime}\right) J_{b}\left(\alpha^{\prime}\right) \exp [i(b-a) \theta]}{a \Omega_{e}-\Omega-k_{\|} v_{\|}+\omega}\right] f_{0 e} d \vec{v} .
\end{aligned}
$$

\section{The Nonlinear Dispersion Relation:}

The nonlinear dielectric function for high-frequency Bernstein mode wave can be obtained from Poisson's equation

$$
\nabla \cdot \delta \vec{E}_{h}=-4 \pi e n_{e} \int\left[\delta f_{h}(\vec{K}, \Omega)+\Delta f(\vec{K}, \Omega)\right] d \vec{v} .
$$

This takes the form

$$
\delta E_{h}(\vec{K}, \Omega) \epsilon_{h}(\vec{K}, \Omega)=0
$$


The dispersion relation thus obtain can be written as

$$
\epsilon_{h}(\vec{K}, \Omega)=\epsilon_{0}(\vec{K}, \Omega)+\epsilon_{d}(\vec{K}, \Omega)+\epsilon_{p}(\vec{K}, \Omega)
$$

where $\epsilon_{0}(\vec{K}, \Omega)$ is the linear part, $\epsilon_{d}(\vec{K}, \Omega)$ is the direct coupling part and $\epsilon_{p}(\vec{K}, \Omega)$ is the polarization coupling part, The expressions of these parts are given by

$$
\begin{aligned}
& \epsilon_{0}(\vec{K}, \Omega)=+\left(\frac{\omega_{p e}^{2}}{K_{\perp}^{2}}\right)\left(\frac{m}{T_{e}}\right) \int\left\{1+\left(\Omega-\frac{\epsilon^{\prime} T_{e} K_{\perp}}{m \Omega_{e}}\right)\right. \\
&\left.\times \sum_{a, b} \frac{J_{a}(\alpha) J_{b}(\alpha) \exp \{i(b-a) \theta\}}{a \Omega_{e}-\Omega}\right\} d \vec{v} . \\
& \cdot \\
& \epsilon_{d}(\vec{K}, \Omega)=-\left(\frac{\omega_{p e}}{K_{\perp}}\right)^{2}\left(\frac{e}{m}\right)^{2} \int\left[\frac { m } { T _ { e } } \left(1+\left\{\Omega-\frac{\epsilon^{\prime} T_{e} K_{\perp}}{m \Omega_{e}}\right\} \times\right.\right. \\
&\left.\sum_{a, b} \frac{J_{a}(\alpha) J_{b}(\alpha) \exp \{i(b-a) \theta\}}{a \Omega_{e}-\Omega}\right) \times \\
&\left\{\frac { E _ { l \perp } } { K _ { \perp } } \frac { m } { T _ { e } } \left(1+\left\{\Omega-\omega+k_{\|} v_{\|}-\frac{\epsilon^{\prime} T_{e} K_{\perp}}{m \Omega_{e}}\right\} \times\right.\right. \\
&\left.\sum_{p, q} \frac{J_{p}\left(\alpha^{\prime}\right) J_{q}\left(\alpha^{\prime}\right) \exp \{i(q-p) \theta\}}{p \Omega_{e}-\Omega-k_{\|} v_{\|}+\omega}\right) \\
&\left.-E_{l \|} \frac{\partial}{\partial v_{\|}} \sum_{p, q} \frac{J_{p}\left(\alpha^{\prime}\right) J_{q}\left(\alpha^{\prime}\right) \exp \{i(q-p) \theta\}}{p \Omega_{e}-\Omega-k_{\|} v_{\|}+\omega}\right\}+ \\
& \frac{m}{T_{e}}\left(\frac{E_{l \|} \frac{\partial}{\partial v_{\|}} f_{0 e}}{-\omega+k_{\|} v_{\|}+i 0}\right)\left(1+\left\{\Omega-\omega+k_{\|} v_{\|}-\right.\right. \\
&\left.\left.\left.\frac{\epsilon^{\prime} T_{e} K_{\perp}}{m \Omega_{e}}\right\} \sum_{p, q} \frac{J_{p}\left(\alpha^{\prime}\right) J_{q}\left(\alpha^{\prime}\right) \exp \{i(q-p) \theta\}}{p \Omega_{e}-\Omega-k_{\|} v_{\|}+\omega}\right)\right] \times \\
& {\left[\frac{E_{l \perp}}{K_{\perp}} \frac{m}{T_{e}}(1+\{\Omega\right.} \\
&\left.\sum_{s, t} \frac{J_{s}(\alpha) J_{t}(\alpha) \exp [i(t-s) \theta]}{s \Omega_{e}-\Omega} \sum_{s, t} \frac{\epsilon_{s}(\alpha) T_{e}(\alpha) \exp [i(t-s) \theta]}{m \Omega_{\perp}}\right\} \times \\
& s \Omega_{e}-\Omega \\
& \hline
\end{aligned}
$$


The expression for $\epsilon_{p}(\vec{K}, \Omega)$ is very bulky, we can write it in the form

$$
\epsilon_{p}(\vec{K}, \Omega)=\frac{\omega_{p e}^{4}}{K_{\perp}^{2}|\vec{K}-\vec{k}|^{2} R}\left(\frac{e}{m}\right)^{2}[(A+B) \times(C+D)] .
$$

where A,B,C,D are given by

$$
\begin{aligned}
A= & -\int\left[\frac { m } { T _ { e } } \left\{1+\left(\Omega-\omega-k_{\|} v_{\|}-\frac{\epsilon^{\prime} T_{e} K_{\perp}}{m \Omega_{e}}\right) \times\right.\right. \\
& \left.\sum_{a, b} \frac{J_{a}\left(\alpha^{\prime}\right) J_{b}\left(\alpha^{\prime}\right) \exp \{i(b-a) \theta\}}{a \Omega_{e}-\Omega-k_{\|} v_{\|}+\omega}\right\}-k_{\|} \frac{\partial}{\partial v_{\|}} \times \\
& \left.\sum_{a, b} \frac{J_{a}\left(\alpha^{\prime}\right) J_{b}\left(\alpha^{\prime}\right) \exp \{i(q-p) \theta\}}{a \Omega_{e}-\Omega-k_{\|} v_{\|}+\omega}\right] \times\left[\frac{E_{l \perp}}{K_{\perp}} \frac{m}{T_{e}}(1+\right. \\
& \left.\left\{\Omega-\frac{\epsilon^{\prime} T_{e} K_{\perp}}{m \Omega_{e}}\right\} \sum_{s, t} \frac{J_{s}(\alpha) J_{t}(\alpha) \exp \{i(t-s) \theta\}}{s \Omega_{e}-\Omega}\right) \\
& \left.+E_{l \|} \frac{\partial}{\partial v_{\|}} \sum_{s, t} \frac{J_{s}(\alpha) J_{t}(\alpha) \exp \{i(t-s) \theta\}}{s \Omega_{e}-\Omega}\right] f_{0 e} d \vec{v} .
\end{aligned}
$$

$$
\begin{aligned}
B= & \int\left[\frac { m } { T _ { e } } \left(1+\left\{\Omega-\frac{\epsilon^{\prime} T_{e} K_{\perp}}{m \Omega_{e}}\right\}\right.\right. \\
& \left.\sum_{a, b} \frac{J_{a}(\alpha) J_{b}(\alpha) \exp \{i(b-a) \theta\}}{a \Omega_{e}-\Omega}\right)-k_{\|} \frac{\partial}{\partial v_{\|}} \times \\
& \left.\sum_{a, b} \frac{J_{a}(\alpha) J_{b}(\alpha) \exp \{i(b-a) \theta\}}{a \Omega_{e}-\Omega}\right]\left(\frac{\frac{\partial}{\partial v_{\|}} f_{o e}}{\omega-k_{\|} v_{\|}+i 0}\right) d \vec{v} .
\end{aligned}
$$




$$
\begin{aligned}
C= & \int\left[\frac { m } { T _ { e } } \left(1+\left\{\Omega-\frac{\epsilon^{\prime} T_{e} K_{\perp}}{m \Omega_{e}}\right\} \times\right.\right. \\
& \left.\left.\sum_{a, b} \frac{J_{a}(\alpha) J_{b}(\alpha) \exp [i(b-a) \theta]}{a \Omega_{e}-\Omega}\right)\right] \times \\
& {\left[\frac { E _ { l \perp } } { K _ { \perp } } \frac { m } { T _ { e } } \left(1+\left\{\Omega-\omega+k_{\|} v_{\|}-\frac{\epsilon^{\prime} T_{e} K_{\perp}}{m \Omega_{e}}\right\} \times\right.\right.} \\
& \left.\sum_{p, q} \frac{J_{p}\left(\alpha^{\prime}\right) J_{q}\left(\alpha^{\prime}\right) \exp \{i(q-p) \theta\}}{p \Omega_{e}-\Omega+k_{\|} v_{\|}+\omega}\right)+ \\
& \left.E_{l \|} \frac{\partial}{\partial v_{\|}} \sum_{p, q} \frac{J_{p}\left(\alpha^{\prime}\right) J_{q}\left(\alpha^{\prime}\right) \exp \{i(q-p) \theta\}}{p \Omega_{e}-\Omega+k_{\|} v_{\|}+\omega}\right] f_{0 e} d \vec{v} . \\
& D=-\int \frac{m}{T_{e}}\left(\frac{\frac{\partial}{\partial v_{\|}} f_{o e}}{-\omega+k_{\|} v_{\|}+i 0}\right) \times \\
& \left(1+\left\{\Omega-\omega+k_{\|} v_{\|}-\frac{\epsilon^{\prime} T_{e} K_{\perp}}{m \Omega_{e}}\right\} \times\right. \\
& \left.\sum_{p, q} \frac{J_{p}\left(\alpha^{\prime}\right) J_{q}\left(\alpha^{\prime}\right) \exp \{i(q-p) \theta\}}{p \Omega_{e}-\Omega-k_{\|} v_{\|}+\omega}\right) d \vec{v} .
\end{aligned}
$$

\section{Instability:-}

The growth rate of Bernstein mode wave is calculated by using the following formula:

$$
\frac{\gamma_{h}}{\Omega}=-\left[\frac{I_{m} \epsilon_{h}+\frac{1}{2} \frac{\partial^{2} \epsilon_{0}}{\partial \Omega \partial t}}{\Omega \frac{\partial \epsilon_{0}}{\partial \Omega}}\right]_{\Omega_{r}} .
$$

The second part of growth rate formula (Eq.(27)) is due to reverse absorption effect which in our case is

$$
\begin{aligned}
\frac{\partial^{2} \epsilon_{0}}{\partial \Omega \partial t}= & \left(\frac{\omega_{p e}^{2}}{K_{\perp}^{2}}\right)\left(\frac{m}{T_{e}}\right) \int\left[\sum_{a, b} \frac{J_{a}(\alpha) J_{b}(\alpha) \exp \{i(b-a) \theta\}}{a \Omega_{e}-\Omega}\right. \\
& \left.\times\left\{1+\left(\Omega-\frac{\epsilon^{\prime} T_{e} K_{\perp}}{m \Omega_{e}}\right) \frac{1}{a \Omega-\Omega}\right\} \frac{\partial f_{0 e}}{\partial t}\right] d \vec{v} .
\end{aligned}
$$

where the slow time change of electron distribution function is given by

$$
\frac{\partial f_{0 e}\left(v_{\|}\right)}{\partial t}=\pi\left(\frac{e}{m}\right)^{2} \frac{\partial}{\partial v_{\|}} \sum_{\vec{k}, \omega}\left|E_{l \|}(\vec{k})\right|^{2} \delta\left(\omega-k_{\|} v_{\|}\right) \frac{\partial f_{0 e}}{\partial v_{\|}} .
$$


After partial integration this contribution due to the reverse absorption effect becomes zero.

We consider the plasma-maser interaction between Bernstein mode wave and drift wave turbulence. The condition for the plasma-maser is $\omega=k_{\|} v_{\|}$. We first estimate the linear part of the dielectric function of Bernstein mode wave from Eq.(20), and considering the fact that for Bernstein mode wave the most dominant contribution to Bessel's sum comes from the term $\mathrm{a}=\mathrm{s}=\mathrm{p}=1$,

$$
\begin{aligned}
\epsilon_{0}(\vec{K}, \Omega)=1 & +\left(\frac{\omega_{p e}^{2}}{K_{\perp}^{2}}\right)\left(\frac{m}{T_{e}}\right)+\left(\frac{\omega_{p e}^{2}}{2 \Omega_{e}^{2}}\right) \times \\
& \left\{\frac{\Omega}{\Omega_{e}-\Omega}-\frac{\epsilon^{\prime} T_{e} K_{\perp}}{m \Omega_{e} \Omega} \times \frac{1}{\Omega_{e}-\Omega}\right\} .
\end{aligned}
$$

From Eq.(30),we obtain,

$$
\frac{\partial \epsilon_{0}}{\partial \Omega}=\left(\frac{\omega_{p e}^{2}}{K_{\perp}^{2}}\right) \times \frac{1}{\left(\Omega_{e}-\Omega\right)^{2}} \times\left(\Omega-\frac{\epsilon^{\prime} T_{e} K_{\perp}}{m \Omega_{e}}\right) .
$$

The dominant contribution, for a magnetized plasma, to the plasma-maser comes from the imaginary part of polarization coupling term. So we calculate the $I_{m} \epsilon_{p}(\vec{K}, \Omega)$ from Eq.(22). We observe that $\mathrm{A}$ and $\mathrm{C}$ are real and $B \propto D^{\star}$, where $\star$ denotes complex conjugate. So we have

$$
I_{m} \epsilon_{p}(\vec{K}, \Omega)=\frac{\omega_{p e}^{4}}{K_{\perp}^{2}|\vec{K}-\vec{k}|^{2} R}\left(\frac{e}{m}\right)^{2}\left[A \times I_{m} D+C \times I_{m} B\right] .
$$

Here we consider the dominant part which comes from $A \times I_{m} D$. 
Hence from Eq. (23), by applying Plasma-maser condition, we have

$$
\begin{aligned}
A= & -\int\left[\frac{m}{T_{e}}\left\{1+\left(\Omega-\frac{\epsilon^{\prime} T_{e} K_{\perp}}{m \Omega_{e}}\right) \frac{J_{1}^{2}\left(\alpha^{\prime}\right)}{\Omega_{e}-\Omega}\right\}-k_{\|} \frac{\partial}{\partial v_{\|}} \times\right. \\
& \left.\frac{J_{1}^{2}\left(\alpha^{\prime}\right)}{\Omega_{e}-\Omega}\right] \times\left[\frac { E _ { l \perp } } { K _ { \perp } } \frac { m } { T _ { e } } \left\{1+\left(\Omega-\frac{\epsilon^{\prime} T_{e} K_{\perp}}{m \Omega_{e}}\right) \times\right.\right. \\
& \left.\left.\frac{J_{1}^{2}(\alpha)}{\Omega_{e}-\Omega}\right\} f_{0 e}(\vec{v})-E_{l \|} \frac{\partial}{\partial v_{\|}} f_{0 e}(\vec{v}) \frac{J_{1}^{2}(\alpha)}{\Omega_{e}-\Omega}\right] d \vec{v} \\
= & \left(\frac{m}{T_{e}}\right)^{2} \frac{E_{l \perp}}{K_{\perp}} \int\left[1+\left(\Omega-\frac{\epsilon^{\prime} T_{e} K_{\perp}}{m \Omega_{e}}\right) \frac{J_{1}^{2}\left(\alpha^{\prime}\right)}{\Omega_{e}-\Omega}+\right. \\
& \left(\Omega-\frac{\epsilon^{\prime} T_{e} K_{\perp}}{m \Omega_{e}}\right) \frac{J_{1}^{2}\left(\alpha^{\prime}\right)}{\Omega_{e}-\Omega}+\left(\Omega-\frac{\epsilon^{\prime} T_{e} K_{\perp}}{m \Omega_{e}}\right) \times \\
& \left.\frac{J_{1}^{2}\left(\alpha^{\prime}\right) J_{1}^{2}(\alpha)}{\left(\Omega_{e}-\Omega\right)^{2}}\right] 2 \pi v_{\perp} f_{0 e}\left(v_{\perp}\right) d v_{\|} d v_{\perp} \\
= & \left(\frac{m}{T_{e}}\right)^{2} \frac{E_{l \perp}}{K_{\perp}}\left[\int_{-\infty}^{\infty} f_{0 e}\left(v_{\|}\right) d v_{\|} \times \int_{-\infty}^{0} 2 \pi v_{\perp} f_{0 e}\left(v_{\perp}\right) d v_{\perp}+\right. \\
& \left(\Omega-\frac{\epsilon^{\prime} T_{e} K_{\perp}}{m \Omega_{e}}\right) /\left(\Omega_{e}-\Omega\right) \int_{-\infty}^{\infty} f_{0 e}\left(v_{\|}\right) d v_{\|} \times \\
& \left\{\int_{-\infty}^{0} 2 \pi J_{1}^{2}(\alpha) v_{\perp} f_{0 e}\left(v_{\perp}\right) d v_{\perp}+\int_{-\infty}^{0} 2 \pi J_{1}^{2}\left(\alpha^{\prime}\right) v_{\perp} \times\right. \\
& \left.\left.f_{0 e}\left(v_{\perp}\right) d v_{\perp}+\int_{-\infty}^{0} 2 \pi J_{1}^{2}\left(\alpha^{\prime}\right) J_{1}^{2}(\alpha) v_{\perp} f_{0 e}\left(v_{\perp}\right) d v_{\perp}\right\}\right] \\
= & \left(\frac{E_{l \perp}}{K_{\perp}}\right)\left(\frac{m}{T_{e}}\right)\left\{\frac{\Omega-\left(\epsilon^{\prime} T_{e} K_{\perp} / m \Omega_{e}\right)}{\Omega_{e}-\Omega} \frac{\left(K_{\perp}-k_{\perp}\right)^{2}}{2 \Omega_{e}^{2}} .\right.
\end{aligned}
$$

after simplification and taking dominating terms only, considering $J_{1}^{2}\left(\alpha^{\prime}\right) \sim J_{1}^{2}(\alpha)$ 


$$
\begin{aligned}
& I_{m} D=\int_{-\infty}^{0} \int_{-\infty}^{\infty} \frac{m}{T_{e}} I_{m}\left(\frac{E_{l \|} \frac{\partial}{\partial v_{\|}} f_{o e}\left(v_{\|}\right)}{-\omega+k_{\|} v_{\|}+i 0}\right)(1+\{\Omega-\omega+ \\
& \left.\left.k_{\|} v_{\|}-\frac{\epsilon^{\prime} T_{e} K_{\perp}}{m \Omega_{e}}\right\} \sum_{p, q} \frac{J_{p}\left(\alpha^{\prime}\right) J_{q}\left(\alpha^{\prime}\right) \exp \{i(q-p) \theta\}}{p \Omega_{e}-\Omega-k_{\|} v_{\|}-\omega}\right) \times \\
& 2 \pi v_{\perp} f_{0 e}\left(v_{\perp}\right) d v_{\|} d v_{\perp} \\
& =\frac{m}{T_{e}} \int_{-\infty}^{0} \int_{-\infty}^{\infty}\left\{1+\left(\Omega-\frac{\epsilon^{\prime} T_{e} K_{\perp}}{m \Omega_{e}}\right) \frac{J_{1}^{2}\left(\alpha^{\prime}\right)}{\Omega_{e}-\Omega}\right\} \times \\
& I_{m}\left(\frac{E_{l \|} \frac{\partial}{\partial v_{\|}} f_{o e}\left(v_{\|}\right)}{-\omega+k_{\|} v_{\|}+i 0}\right) d v_{\|} 2 \pi v_{\perp} f_{0 e}\left(v_{\perp}\right) d v_{\|} d v_{\perp} \\
& =\frac{m}{T_{e}}\left[\int_{-\infty}^{0} 2 \pi v_{\perp} f_{0 e}\left(v_{\perp}\right) d v_{\perp}+\left\{\frac{\Omega-\left(\epsilon^{\prime} T_{e} K_{\perp} / m \Omega_{e}\right)}{\Omega_{e}-\Omega}\right\} \times\right. \\
& \left.\int_{-\infty}^{0} 2 \pi v_{\perp} J_{1}^{2}\left(\alpha^{\prime}\right) f_{0 e}\left(v_{\perp}\right) d v_{\perp}\right] \times \\
& \int_{-\infty}^{\infty} I_{m}\left(\frac{E_{l \|} \frac{\partial}{\partial v_{\|}} f_{o e}\left(v_{\|}\right)}{-\omega+k_{\|} v_{\|}+i 0}\right) d v_{\|} \\
& =E_{l \|}\left\{\frac{\Omega-\left(\epsilon^{\prime} T_{e} K_{\perp} / m \Omega_{e}\right)}{\Omega_{e}-\Omega}\right\} \frac{\left(K_{\perp}-k_{\perp}\right)^{2}}{2 \Omega_{e}^{2}} \times \\
& \frac{2 \sqrt{\pi}}{v_{e}^{3}} \frac{\omega}{k_{\|}\left|k_{\|}\right|} \exp \left\{-\left(\frac{\omega}{k_{\|} v_{e}}\right)^{2}\right\} \text {. }
\end{aligned}
$$

after simplification and taking dominating terms only.

Using the expressions for $A$ and $I_{m} D$ in Eq.(32), we get

$$
\begin{aligned}
I_{m} \epsilon_{p}(\vec{K}, \Omega)= & -\left(\frac{\omega_{p e}^{4}}{K_{\perp}^{2}}\right)\left(\frac{e}{m}\right)^{2}\left(\frac{E_{l \perp}}{K_{\perp}}\right)\left(\frac{m}{T_{e}}\right) \times \\
& \left\{\frac{\Omega-\left(\epsilon^{\prime} T_{e} K_{\perp} / m \Omega_{e}\right)}{\Omega_{e}-\Omega}\right\} \frac{\left(K_{\perp}-k_{\perp}\right)^{2}}{2 \Omega_{e}^{2}} E_{l \|} \times \\
& \left\{\frac{\Omega-\left(\epsilon^{\prime} T_{e} K_{\perp} / m \Omega_{e}\right)}{\Omega_{e}-\Omega}\right\} \frac{\left(K_{\perp}-k_{\perp}\right)^{2}}{2 \Omega_{e}^{2}} \times \\
& \frac{2 \sqrt{\pi}}{v_{e}^{3}} \frac{\omega}{k_{\|}\left|k_{\|}\right|} \exp \left\{-\left(\frac{\omega}{k_{\|} v_{e}}\right)^{2}\right\} .
\end{aligned}
$$

Here we use the amplitude ratio of the electric field as derived by Nambu et.al.[21] 


$$
\frac{E_{l \|}(\vec{k})}{E_{l \perp}(\vec{k})}=\frac{k_{\|}}{k_{\perp}} \frac{T_{e}}{T_{i}}\left\{1-I_{0}\left(\beta_{i}\right) \exp \left(\beta_{i}\right)\right\} \equiv-\left(\frac{k_{\|}}{k_{\perp}}\right) Q^{-1}
$$

where $T_{e}, T_{i}$ and $I_{0}$ are the electron, ion temperature and modified Bessel function respectively.

$$
\beta_{i}=\left(k_{\perp} \sigma_{i}\right)^{2} / 2, Q=\left(\frac{T_{e}}{T_{i}}\right)\left\{1-I_{0}\left(\beta_{i}\right) \exp \left(\beta_{i}\right)\right\}^{-1} \text { and } \sigma_{i} \text { is the ion gyroradius. }
$$

The growth rate of Bernstein mode wave due to polarization coupling term, retaining the dominating terms only, is calculated by using Eq.(27) as

$$
\begin{aligned}
\frac{\gamma_{p}}{\Omega}= & \left(\frac{\omega_{p e}}{\Omega_{e}}\right)^{2}\left|E_{l \perp}(\vec{k})\right|^{2}\left\{\Omega-\frac{\epsilon^{\prime} T_{e} K_{\perp}}{m \Omega_{e}}\right\} \frac{K_{\perp}}{v_{e}} \frac{k_{\|}}{k_{\perp}} \times \\
& \sqrt{\frac{\pi m}{M}} Q^{-1} \exp \left\{-\left(\frac{\omega}{k_{\|} v_{e}}\right)^{2}\right\}
\end{aligned}
$$

In obtaining $R|\vec{K}-\vec{k}|^{2}$, we expand $R$, from Eq.(18), about small argument $\vec{k}$ and $\omega$ in $R$ and we have used the relation $\epsilon_{0}(\vec{K}, \Omega)=0$. To the lowest order approximation we can estimate $R|\vec{K}-\vec{k}|^{2} \simeq k_{\|}^{2}$

\section{Discussion :}

Earlier investigation [25] on interaction among drift wave and high frequency Trivelpiece - Gould mode through plasma maser effect, interesting result about the stability of drift mode in homogeneous plasma were predicted. In this paper, it has been shown that nonlinear damping of drift mode occurs through energy exchange among drift mode and electrostatic Trivelpiece-Gould mode. Here the nonlinear dispersion relation of ion acoustic mode in presence of drift wave turbulence field is evaluated, which is a common feature of inhomogeneous plasma.

Historically, the plasma-maser from the direct coupling term $\epsilon_{d}(\vec{k}, \Omega)$ was pointed by Tystovich and co-worker [26] for an ion-sound turbulence in unmagnetized plasma.But in a closed system the plasma maser contribution from direct coupling part exactly cancels out with the reverse absorption effect [27], i.e.

$$
I_{m} \epsilon_{d}(\vec{K}, \Omega)+\frac{1}{2} \frac{\partial^{2} \epsilon_{0}\left(\vec{K}, \Omega, f_{0 e}(t)\right)}{\partial \Omega \partial t}=0 .
$$

In this system the low-frequency turbulence and the background electron distribution function are not fixed by external agents, but are free to evolve self-consistently to form a quasilinear plateau. Then both the plasma maser and the reverse process due to quasilinear effect coexist and there is no net growth of the nonresonant high-frequency test wave. 
In an open system, with a particle supply from outside, the electron distribution function is fixed by external agents and the reversed absorption effect vanishes

$$
\partial^{2} \epsilon_{0}\left(\vec{K}, \Omega, f_{0 e}(t)\right) /(\partial \Omega \partial t)=0
$$

then the stationary state without quasilinear plateau is possible. Accordingly, the energy transferred from the low-frequency waves by resonant interaction must go into an unstable high-frequency mode. This type of enhanced radiation process is termed as dissipative structure in plasma turbulence [27]. The dissipation is due to resonant wave-particle collisionless heating. The nonresonant wave amplification or absorption can be due to perturbation of resonant wave-particle collisionless interaction.

Here, an attempt has been made to consider the plasma maser effect among electrons, resonant mode drift wave and the nonresonant Bernstein mode wave in inhomogeneous magnetized plasma. The inhomogeneity feature of the plasma considered here provides an additional source of free energy to the system and is now important to clarify the role of free energy of these drifting particles in this particular mode-mode coupling process of turbulent inhomogeneous plasma. The nonlinear dispersion relation Eq.(19) is obtained by neglecting the gradients of the confining magnetic field. Since polarization coupling is the dominating term in the Plasma maser effect, the growth rate of Bernstein mode wave is estimated from polarization coupling term only.

When there is no density gradient $\left(\epsilon^{\prime}=0\right)$ the growth rate of Bernstein mode for homogeneous plasma is obtained,

from Eq.(34), as

$$
\frac{\gamma_{p}}{\Omega}=\left(\frac{\omega_{p e}}{\Omega_{e}}\right)^{2}\left|E_{l \perp}(\vec{k})\right|^{2} \frac{K_{\perp}}{v_{e}} \frac{k_{\|}}{k_{\perp}} \sqrt{\frac{\pi m}{M}} \frac{\Omega}{Q} .
$$

When high density gradient is present in the system of plasma then the growth rate of Bernstein mode is estimated,

from Eq.(34), as

$$
\frac{\gamma_{p}}{\Omega}=\left(\frac{\omega_{p e}}{\Omega_{e}}\right)^{2}\left|E_{l \perp}(\vec{k})\right|^{2} \frac{v_{e}}{\Omega_{e}} \frac{k_{\|}}{k_{\perp}} \sqrt{\frac{\pi m}{M}} Q^{-1} \times \epsilon^{\prime} .
$$

However for small order gradient there is no effect in the growth rate and Eq.(35) will give the estimate for such small gradient situation.

By using the following observational data in space: $\quad E_{l \perp} \sim 50 \mu v m^{-1}, \quad \Omega \sim \omega_{p e} \sim$ $100 \mathrm{KHz}, \quad k_{\|} \sim 2 \pi \times 10^{-7} \mathrm{~cm}^{-1}, K_{\perp} \sim 2 \pi \times 10^{-5} \mathrm{~cm}^{-1}, \Omega_{e} \sim 250 \mathrm{KHz}$, and $\sqrt{\frac{m}{M}} \sim 43$

We have from Eq.(35)

$$
\frac{\gamma_{p}}{\Omega} \simeq 10^{-1}
$$


and from Eq. (36), we have

$$
\frac{\gamma_{p}}{\Omega} \simeq 10^{-2} \epsilon^{\prime}
$$

We have from Eq.(36)

$$
\frac{\gamma_{p}}{\Omega} \simeq 10^{-1}
$$

taking $\epsilon^{\prime}=10$.

The Plasma-maser instability of electrostatic Bernstein mode wave in the presence of drift wave turbulence driven by a weak electron beam is considered for inhomogeneous plasma. It is found that the Bernstein mode waves propagating perpendicular to a magnetic field are destabilized by the plasma-maser process. Moreover, the density gradient influences the growth rate of Bernstein mode wave. The theory agrees with the recent experiments in astrophysical and space plasma and laboratory and the results have potential importance in the interpretation of anomalous radiation phenomena in astrophysical, laboratory and space plasma.

\section{References :}

[1] : N. D. Angelo and R. W. Motely, Phys. Fluids, 6,422 (1963).

[2] : F. F. Chen, Phy. Rev. Lett. 15, 381 (1965).

[3] : Weiland, Collective Modes in Inhomogeneous Plasma,

Institute of Physics Publishing, Bristol, (2000).

[4] : C. S. Wu and C. L. Lee, Astrophysics, J. 230, 621 - 626 (1979).

[5] : L. Queau, D. R. Pellat and A. Roux, Ann. Geophys. 3, 273 - 291, 1985.

[6] : D. Sundkvist, V. Kranoselskikh, P. K. Shukla, A. Vaivads, M. Andre,

S. Buchert and H. Reme ( London ) 436, 825 ( 2005 ).

[7] : M. Singh and P. N. Deka, Phys. Plasma 12, 102304 ( 2005 ).

[8] : P. N. Deka and A. Borgohain, Phys. Plasmas 18, 042311 ( 2011 ).

[9] : F. Jenko, Phys. Plasmas 7, 515 ( 2000 ).

[10]: M. Nambu, Laser and Particle beams 1, 427 (1986).

[11]: M. Nambu, Space Sci. Rev. 44, 357 (1986).

[12]: S. V. Vladimirov, and M. Y. Yu, Physica Scripta T 113, 32 (2004).

[13]: W. Horton, Rev. Mod. Phys. 71,735 (1999).

[14]: J. C. Perez, W. Horton, K. Gentle, W. L. Rowan, K. Lee and

R. Dahlburg, Phys. Plasmas, 13, 032101 (2006) .

[15]: P. N. Deka, K. S. Goswami and S. Bujarbarua, Planet.

Space. Sci. Vol.45 No.11, 1443 (1997) .

[16] : B. J. Saikia, P. N. Deka and S. Bujarbarua, Contrib.

Plasma Phys. 35,263 (1995).

[17]: M. Singh, Planetary and Space Science 55, 467474 (2007) . 
[18]: M. Singh, Physica Scripta, 74, 55 (2006) .

[19] : M. Singh and P. N. Deka, Pramana, 66, No.3 (2006) .

[20]: N. A. Krall, and A. W. Trivelpiece, Principles of

Plasma Physics, Mc.Graw Hill, New York, (1973).

[21]: M. Nambu, Phys. Fluids, 19, 412 (1976).

[22] : B. T. Tsurutani and G. S. Lakhina, Rev. Geophys.

35, 491, doi: 10,1029/97RG02200 (1997).

[23]: T. H. Stix, Waves in Plasma, American Institute of Physics, New York, 390 (1992).

[24] : M. Nambu, Phys. Fluids 19, 412 (1976).

[25] : M. Nambu, Phys. Rev. A, 35, 1953 (1987).

[26]: V. N. Tystovich, L. Stenflo and H. Wilhelmsson,

Phys. Scripta 11, 251 (1975).

[27]: M. Nambu, T. Hada, S. N. Sarma, and S. Bujarbarua,

J. Phys. Socs. Japan 60, 3004 (1991). 